Sea: evidence from sediment traps. In Upwelling Systems: Evolution Since the Early Miocene (eds Summerhays, C. P., Prell, W. L. and Emeis, K. C.), Geological Society Special Publication, 1992, vol. 64, pp. 93-106.

35. Emeis, K. C., Anderson, D. M., Doose, H., Kroon, D. and SchulzBull, D., Sea surface temperatures and history of monsoon upwelling in the northwest Arabian Sea during the last 500,000 years. Quaternary Res., 1995, 43, 355-361.

36. Ishikawa, S. and Oda, M., Reconstruction of Indian monsoon variability over the past 230,000 years: planktic foraminiferal evidence from the NW Arabian Sea open ocean upwelling area. Mar. Micropaleontol., 2007, 63, 143-154.

37. Ziegler, M. L., Lourens, L. J., Tuenter, E. and Reichart, G. J., High Arabian Sea productivity conditions during MIS 13 -odd monsoon event or intensified overturning circulation at the end of the Mid-Pleistocene transition? Climate Past, 2010, 6, 63-76.

38. Be', A. W. H. and Hutson, W. H., Ecology of planktonic foraminifera and biogeographic patterns of life and fossil assemblages in the Indian Ocean. Micropaleontology, 1977, 23, 369-414.

39. Hemleben, C., Spindler, M. and Anderson, O. R., Modern Planktic Foraminifera, Springer-Verlag, New York, USA, 1989, p. 363.

40. Hilbrecht, H., Morphologic gradation and ecology in Neogloboquadrina pachyderma and $N$. dutertrei (planktic foraminifera) from core top sediments. Mar. Micropaleontol., 1997, 31, 31-43.

41. Schiebel, R., Waniek, J., Bork, M. and Hemleben, C., Planktic foraminiferal production stimulated by chlorophyll redistribution and entrainment of nutrients. Deep-Sea Res. I, 2001, 48, 721-740.

42. Veldhuis, M. J. W., Kraay, G. W., van Bleijswijk, J. D. L. and Baars, M. A., Seasonal and spatial variability in phytoplankton biomass, productivity and growth in the Indian Ocean: the southwest and northeast monsoon, 1992-1993. Deep-Sea Res. I, 1997, 44, 425-449.

43. Almogi-Labin, A., Schmiedl, G., Hemleben, C., Siman-Tov, R., Segl, M. and Meischner, D., The influence of the NE winter monsoon on productivity changes in the Gulf of Aden, NW Arabian Sea, during the last $530 \mathrm{ka}$ as recorded by foraminifera. Mar. Micropaleontol., 2000, 40, 295-319.

44. Rogalla, U. and Andruleit, H., Precessional forcing of coccolithophore assemblages in the northern Arabian sea: implications for monsoonal dynamics during the last 200,000 years. Mar. Geol., 2005, 217, 31-48.

45. Saraswat, R. et al., Inconsistent change in surface hydrography of the eastern Arabian Sea during the last four glacial-interglacial intervals. Geol. Mag., 2019; https://doi.org/10.1017/S0016756819001122.

46. Clemens, S. C. and Prell, W. L., A 350,000 year summer-monsoon multi-proxy stack from the Owen Ridge, northern Arabian Sea. Mar. Geol., 2003, 201, 35-51.

47. Witte, U. and Pfannkuche, O., High rates of benthic carbon remineralisation in the abyssal Arabian Sea. Deep Sea Res. II, 2000, 47, 2785-2804.

48. Schmiedl, G. and Leuschner, D. C., Oxygenation changes in the deep western Arabian Sea during the last 190,000 years: productivity versus deep-water circulation. Paleoceanography, 2005, 20, PA2008; doi:10.1029/2004PA001044.

ACKNOWLEDGEMENTS. We thank the Ocean Drilling Program (ODP) for providing core samples for the present work, and the anonymous reviewer for valuable suggestions that helped improve the manuscript. The present work was partially supported by the grant provided to V.K.P. under INSPIRE Fellowship by Department of Science and Technology, New Delhi.

Received 10 January 2020; revised accepted 15 October 2020

doi: $10.18520 / \mathrm{cs} / \mathrm{v} 119 / \mathrm{i} 11 / 1833-1838$

\section{Perindopril improves cardiac function in doxorubicin-induced cardiotoxicity rats}

\author{
Li-juan Shen ${ }^{1}$, Yong-hua Zhou ${ }^{2}$, Jin-gui Wang ${ }^{3}$, \\ Lan $\mathrm{Li}^{4}$ and $\mathrm{Shu} \mathrm{Lu}^{5, *}$
}

${ }^{1}$ Department of ICU, Wuxi Hospital of Traditional Chinese Medicine, Wuxi Affiliated Hospital Nanjing University of Traditional Chinese Medicine, Wuxi 214071, Jiangsu, China

${ }^{2}$ Jiangsu Institute of Parasitic Diseases, Key Laboratory on Technology for Parasitic Disease Prevention and Control, Ministry of Health, Jiangsu Provincial Key Laboratory on Molecular Biology of Parasites, Jiangsu Provincial Key Subject on Parasitic Diseases,

Wuxi 214064, China

${ }^{3}$ Department of Medical Affairs,

${ }^{4}$ Department of Ultrasonography, and

${ }^{5}$ Department of Cardiology, Wuxi Hospital of Traditional Chinese Medicine, Wuxi Affiliated Hospital Nanjing University of Traditional Chinese Medicine, Wuxi 214071, Jiangsu, China

The present study aimed to observe the effect of perindopril on cardiac function in doxorubicininduced cardiotoxicity rats and explore the underlying molecular mechanisms. We constructed a doxorubicin-induced cardiotoxicity rat model $\left(1.0 \mathrm{mg} \mathrm{kg}^{-1}\right.$, biweekly) for six weeks. Rats in the doxorubicininduced cardiotoxicity group exhibited impaired cardiac function, disorganized sarcomeres and increased levels of brain serum natriuretic peptide, creatine kinase isozyme MB and troponin I. In addition, compared to normal hearts, doxorubicin-induced cardiotoxicity hearts exhibited significantly higher levels of angiotensinogen, angiotensin II (Ang II), angiotensin II type 1 receptor, protein kinase $C$, reactive oxygen species (ROS), high mobility group box 1 (HMGB1), nuclear factor kappa $B$, tumour necrosis factor- $\alpha$, interleukin-6 as well as interleukin-1 $\beta$. Positive correlation was found among Ang II, ROS and HMGB1. After treatment with an angiotensin-converting enzyme inhibitor perindopril, cardiac function and inflammation induced by doxorubicin had distinctly improved. Intriguingly, the levels of Ang II, ROS and HMGB1 decreased significantly. Our findings suggest that perindopril improves cardiac function in doxorubicin-induced cardiotoxicity rats, which might be related with Ang II/ROS/HMGB1.

Keywords: Cardiac function, doxorubicin-induced cardiotoxicity, myocardial injury, perindopril, rat model, reactive oxygen species.

DOXORUBICIN as a broad-spectrum antitumour anthracycline derived from Streptomyces, is widely used to treat a variety of cancers ${ }^{1}$. The serious adverse effects of doxorubicin on cardiac toxicity limit its clinical applications $^{2}$. Doxorubicin can promote congestive heart failure when it exceeds the cumulative dose of $400-700 \mathrm{mg} / \mathrm{m}^{2}$

*For correspondence. (e-mail: panda55007@163.com) 
in adults and $300 \mathrm{mg} / \mathrm{m}^{2}$ in children ${ }^{3}$. Chronic cardiotoxicity develops during chemotherapy or within the first year after completion ${ }^{4}$. It is usually characterized by left ventricular systolic dysfunction, thereby progressing to dilated cardiomyopathy as well as chronic heart failure ${ }^{5}$. Despite advanced research over the years, the molecular mechanism of cardiotoxicity caused by doxorubicin is still unclear.

The renin-angiotensin-aldosterone system (RAAS) plays a key role in ventricular remodelling, which is manifested by a significant increase in plasma angiotensin II (Ang II) levels ${ }^{6}$. RAAS over activation along with activation of other neurohormonal systems (e.g. sympathetic nervous system) leads to myocardial dysfunction ${ }^{7}$. Ang II attaches to the angiotensin II type-1 receptor (AT1R), which activates NOX to promote reactive oxygen species (ROS) production in cardiomyocytes and endothelial cells $^{8}$. ROS affect nearly all of the key features of cardiac maladaptation, such as contractile dysfunction, extracellular matrix remodelling and arrhythmia ${ }^{9}$.

Inflammation is widely detected for patients with heart failure, which has been become a predictor for clinical outcomes ${ }^{10}$. High mobility group box 1 (HMGB1) is a key inflammatory mediator and proinflammatory cytokine $^{11}$ that can lead to a variety of inflammatory responses as well as heart failure ${ }^{12-14}$. Moreover, previous studies have found that HMGB1 and its inflammation signalling pathway are highly activated in dilated cardiomyopathy rats, and are associated with the size of the cardiac cavity and cardiac function ${ }^{11}$. Ang II induces increased secretion of HMGB1 and overactivation of Toll-like receptor-4 (TLR4) in hypertensive nephritis, thereby regulating the production of cytokines induced by nuclear factor kappa $\mathrm{B}(\mathrm{NF}-\kappa \mathrm{B})^{15}$. Other studies have reported that oxidative stress modulates the secretion of HMGB1 (refs 16, 17). However, there is no report on whether high HMGB1 expression is caused by the interaction of Ang II and ROS in doxorubicin-induced cardiotoxicity. Based on the above reports, we hypothesize that the relationships between Ang II and ROS promote the secretion of HMGB1 and lead to subsequent inflammatory responses, aggravating ventricular remodelling and myocardial injury.

The present study analyses the effects of perindopril on cardiac function in doxorubicin-induced cardiotoxicity rats and explores potential molecular mechanisms for doxorubicin-induced cardiotoxicity.

Animal protocols were approved by the Animal Care and Use Committee of the Institute of Key Laboratory on Technology for Parasitic Disease Prevention and Control, Ministry of Health, China. Animal assays were in compliance with the Guide for the Care and Use of Laboratory Animals by the Chinese Ministry of Science and Technology.

Totally 60 Sprague-Dawley rats (8-week-old, weighing 260-280 g; Beijing SCXK Laboratory Animal Co
Ltd, Beijing, China) were randomly separated into normal group $(n=20)$, doxorubicin-induced cardiotoxicity group $(n=20)$ and perindopril group $(n=20)$. Rats in the doxorubicin-induced cardiotoxicity and perindopril groups were induced with doxorubicin, as previously reported ${ }^{18}$. Briefly, each rat was intraperitoneally administered $1 \mathrm{mg} \mathrm{kg}^{-1}$ doxorubicin for six weeks, twice a week, and observed for two weeks. At the beginning rats in the perindopril group were administered perindopril (3.0 $\mathrm{mg} \mathrm{kg}^{-1} \mathrm{~d}^{-1}$ ) by gavage ${ }^{7}$, while those in the normal and doxorubicin-induced cardiotoxicity groups were administered the same amount of saline. Finally, all rats were euthanized through the intraperitoneal injection of sodium pentobarbital $\left(200 \mathrm{mg} \mathrm{kg}^{-1}\right)$.

Echocardiography was performed using an ultrasound (Philips CX50, NY, USA) with a $12-\mathrm{MHz}$ transducer. The left ventricular end-diastolic diameter and endsystolic diameter (LVEDD and LVESD respectively) were analysed. The fractional shortening (FS) and ejection fraction (EF) were calculated automatically by the ultrasound software, and echocardiography was used to verify rat death, according to whether its heart was working.

Positron emission tomography (PET) was used to monitor LV dilation in rats, as described previously ${ }^{19,20}$. PET imaging was carried out using a PET scanner (Siemens Invenon P120, Inveon Dedicate, Munich, Germany). Briefly, the rats were sedated with $10 \%$ chloral hydrate (300 $\mathrm{mg} \mathrm{kg}^{-1}$, i.p.) after $8 \mathrm{~h}$ of fasting and water restriction. Following intravenous administration of 2-deoxy-2- $\left({ }^{18} \mathrm{~F}\right)$ fluoro-D-glucose (FDG; $19 \pm 5 \mathrm{MBq}$ ), the electrocardiogram-gated emission was documented for $70 \mathrm{~min}$. Using 7-min transmission scan, myocardial viability was recorded, as previously reported ${ }^{21}$. The following formula was used to define the standard uptake value (SUV): Intensity of radioactivity of the region of interest $\left(\mathrm{MBq} \mathrm{ml}^{-1}\right) /$ Radioactivity intensity of the region of interest (MBq)/Body weight ( $\mathrm{g})$.

ELISA (Blue Base Biological Technology Co Ltd, Shanghai, China) was used to evaluate the serum levels of brain serum natriuretic peptide (BNP), creatine kinase isozyme MB (CKMB), troponin I (TnI), tumour necrosis factor- $\alpha$ (TNF- $\alpha$ ), interleukin-6 (IL-6) as well as interleukin-1 $\beta$ (IL-1 $\beta$ ), according to the manufacturers' instructions.

The Ang II levels in myocardial tissues were measured by radioimmunoassay. A total of $0.2 \mathrm{~g}$ of myocardial tissue was weighed and rinsed several times in physiological saline at $0^{\circ} \mathrm{C}$, and the filter paper was blotted dry. Next, $0.8 \mathrm{ml}$ of cold physiological saline water was added and the tissue was fully ground for the preparation of a $10 \%$ myocardial homogenate (adding anhydrous ethanol, $\quad 0.25 \mathrm{mmol} / \mathrm{l}$ EDTA, $0.34 \mathrm{mmol} / \mathrm{l}$ 8-hydroxyquinoline, $0.32 \mathrm{mmol} / \mathrm{l}$ dimercaptopropanol), according to the manufacturer's specifications (Beijing North Institute of Biotechnology Co Ltd, Beijing, China). An 
enzyme inhibitor was added to each tube. Then the samples were boiled for $10 \mathrm{~min}$ and centrifuged at $1000 \mathrm{~g}$ for $10 \mathrm{~min}$. The supernatant was removed and stored at $-20^{\circ} \mathrm{C}$ until utilization. Ang II levels in myocardial tissues were assessed by radioimmunoassay utilizing the polyclonal antibody 182 (BBP1955, BestBio Science, Shanghai, China). Ang II was radioiodinated $\left({ }^{125} \mathrm{I}\right)$ using the chloramine-T method. Next, the peptides were partially purified through adsorption onto Bond-Elut cartridges. Monoiodinate peptides (specific activity $2176 \mathrm{Ci} / \mathrm{mmol}$ ) were collected employing HPLC chromatography using heptafluorobutyric acid (HFBA)/acetonitrile gradient. Amounts corresponding to 5-10 \#Ci were purified in each HPLC run. The levels of angiotensin peptide standards were determined using amino acid analyses and the values were converted into $\mathrm{pg} / \mathrm{mg}$.

The mRNA levels of AT1R, protein kinase C (PKC), HMGB1 as well as NF- $\kappa \mathrm{B}$ in heart tissues were determined using quantitative PCR with a LightCycler 480 Gene Amplification System (BioER, San Francisco, CA, USA). First, cDNA was synthesized using SYBR ${ }^{\circledR}$ Premix ExTaqTMII (TaKaRa Code: DRR081A). Quantitative measurements were performed using LightCycler (version 1.5) relative quantification software, and the standardization ratios were calculated with the $2^{-\Delta \Delta \mathrm{CT}}$ formula. $\beta$-actin served as a reference ${ }^{22}$. The oligonucleotide sequences were as follows: angiotensinogen: forward, 5'-AAGCAGGCGAGGAGGAGCAG-3', reverse, 5'-GATGGCGAACAGGAACGGACTG-3'; AT1R: forward, 5'-GCTTCAACCTCTACGCCAGTGTG-3', reverse, 5'-CAGCCAGATGATGATGCAGGTGAC-3'; PKC: forward, 5'-GATCGGACGACACGGAATGACTTC-3', reverse, 5'-CATCCACTGGCTGGCATCTTCATC-3'; HMGB 1: forward, 5'-ACCCGGATGCTTCTGTCAAC-3', reverse, 5'-ACAAGAAGGCCGAAGGAGGC-3'; NF- $\kappa$ B: forward, 5'-GCAAACCTGGGAATACTTCATGTGACTAAG-3', reverse, 5'-ATAGGCAAGGTCAGAATGCACCAGAAGTCC- $3^{\prime}$ and $\beta$-actin: forward, 5'-TCCGTAAAGACCTCTATGCCAACA-3', reverse, 5'-GCTAGGAGCCAGGGCAGTAATCT-3'.

The protein levels of HMGB 1 and NF- $\kappa$ B were evaluated using Western blot analysis. Freshly dissected rat hearts were collected for protein extraction and myocardial tissues were lysed via RIPA buffer (Beyotime, Beijing, China) containing a protease inhibitor cocktail. The concentration of protein was detected using a BCA Protein Assay Kit (Bio-Rad, USA). The lysate was loaded onto $12 \%$ (w/v) SDS-PAGE gels. Following electrophoresis, samples were transferred onto PVDF membrane (Millipore, USA), which was then blocked overnight with $5 \%$ skim milk at $4{ }^{\circ} \mathrm{C}$, followed by incubation with rabbit anti-HMGB1 monoclonal antibody (1:600; ab79823, Abcam, UK) and rabbit anti-NF- $\kappa \mathrm{B}$ monoclonal antibody (1: 1000; ab16502, Abcam, UK). The membrane was then incubated for $1 \mathrm{~h}$ with a goat anti-rabbit $\operatorname{IgG}(\mathrm{H}+\mathrm{L})$ horseradish peroxidase (HRP)-labelled secondary anti- body (1 : 10,000; ab205718, Abcam, UK). Finally, the blots were visualized by enhanced chemiluminescence reagents (Co Win Biotech Co Ltd, Beijing, China). AntiGAPDH served as an endogenous control.

The ROS level in myocardial tissue was detected by fluorescence method using dichlorodihydrofluorescein diacetate following the manufacturer's instructions. Following cutting myocardial tissues and ventricular myocytes were trypsin-digested into a single cell suspension. After adjusting the cells to a density of $1 \times 10^{7}$ cells $/ \mathrm{ml}$, dichloro-dihydro-fluorescein diacetate (DCFH-DA) was added and the samples were incubated at $37^{\circ} \mathrm{C}$ for 20 min, washed three times and then treated with enzyme. Finally, the samples were measured by an enzymelabelling instrument.

Hematoxylin and eosin (H\&E) staining was used to examine morphological variations in paraffin-embedded cardiac tissues under a light microscope (Leica DMI6000B, Buffalo Grove, IL, USA). The myocardial ultrastructure was observed using an electron microscopy (JEM-1230, Japan Electronic Co Ltd, Tokyo, Japan).

Data were presented as mean \pm standard deviation (SD). Log-rank (Mantel-Cox) test was used to assess the differences in survival rates among different groups. Comparisons of multiple groups were performed by one-way ANOVA following the Bonferroni post-hoc test. $P<0.05$ was considered significant. All data were analysed using SPSS 21.0 software (CABIT Information Technology, Shanghai, China).

The survival rate of rats in different groups was examined eight weeks after operation. No deaths were reported in the normal group. The injection site had hyperplasia and no exudation following administration of $10 \%$ doxorubicin. However, in the doxorubicin-induced cardiotoxicity group, seven (35\%) rats died from day 14 to day 40. An autopsy revealed that the dead rats exhibited enlarged cardiac chambers, enlarged livers and ascites. In the perindopril group, the cumulative survival rate was

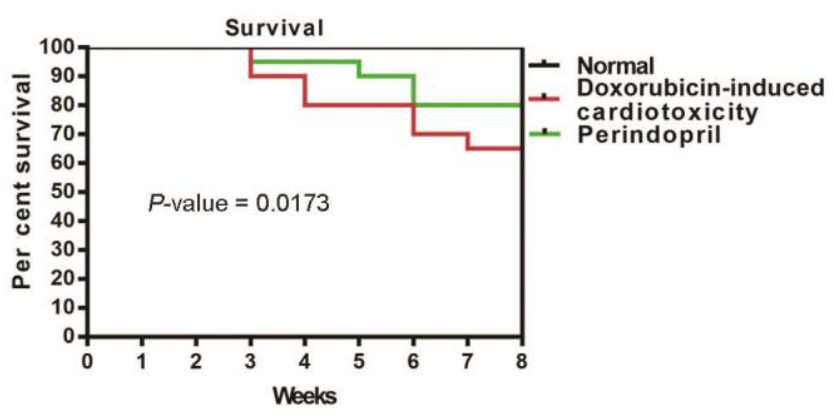

Figure 1. Survival rate of doxorubicin-induced cardiotoxicity rats. The overall survival rate was $65 \%(13 / 20)$ in the doxorubicin-induced cardiotoxicity group (doxorubicin $1.0 \mathrm{mg} \mathrm{kg}^{-1}$ ) and $80 \%(16 / 20)$ in the perindopril group (perindopril $3.0 \mathrm{mg} \mathrm{kg}^{-1} \mathrm{~d}^{-1}$ ). $P$-value calculation method: log-rank (Mantel-Cox) test. Normal: $n=20$; DCM: $n=20$; perindopril: $n=20$. The $P$-value was used to assess any difference between the survival curves of the three groups. 

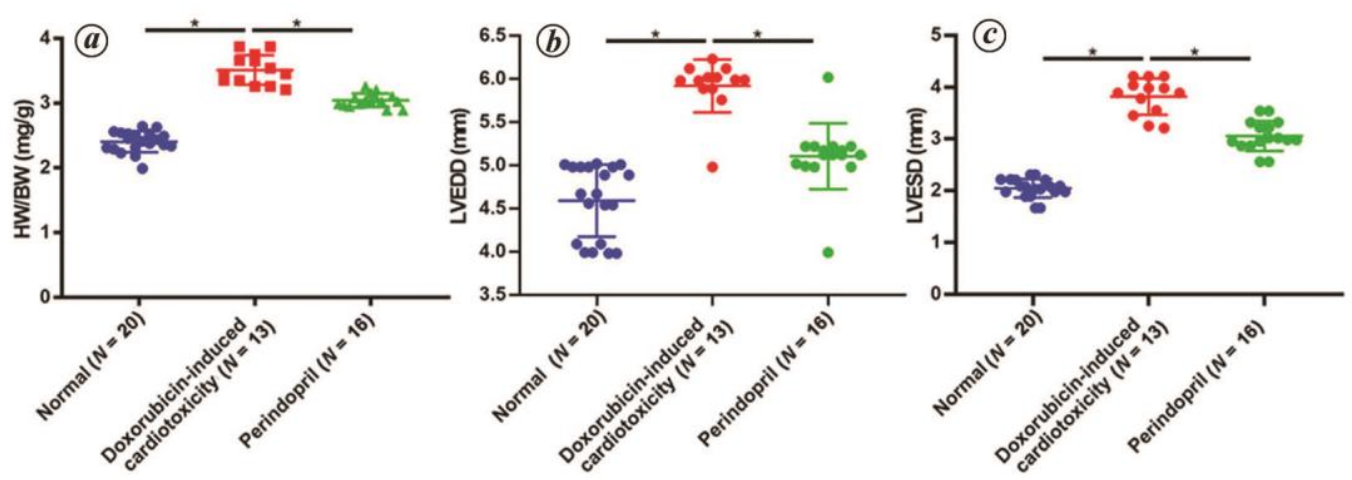

Figure 2. Cardiac structure and echocardiography in doxorubicin-induced cardiotoxicity rats. $\boldsymbol{a}$, The HW/BW ratio in doxorubicin-induced cardiotoxicity rats was markedly increased in comparison to that in the normal rats $(P<0.05)$. The HW/BW ratio in the perindopril group was lower than that in the doxorubicin-induced cardiotoxicity rats $(P<0.05) . \boldsymbol{b}, \boldsymbol{c}$, LVEDD and LVESD levels were distinctly elevated $(P<0.05)$ in the doxorubicin-induced cardiotoxicity rats than in those of the other groups. In comparison to the doxorubicin-induced cardiotoxicity group, the perindopril group exhibited decrease in LVEDD as well as LVESD levels $(P<0.05)$.

$80 \%(16 / 20)$, indicating that perindopril improved the survival of doxorubicin-induced cardiotoxicity rats (Figure 1).

Rats in the normal and doxorubicin-induced cardiotoxicity groups exhibited a significant increase in body weight (BW) during the eight weeks. However, the heart weight $(\mathrm{HW}) / \mathrm{BW}$ ratio in the doxorubicin-induced cardiotoxicity rats was notably elevated in comparison to that in the normal group $(P<0.05)$, and this increase was significantly reduced after perindopril treatment $(P<0.05)$ (Figure $2 a$ ).

Rats in the doxorubicin-induced cardiotoxicity group exhibited markedly increased LVEDD and LVESD levels $(P<0.05)$ (Figure $2 b$ and $c)$ than those in the other two groups, indicating an enlarged cardiac cavity in them. In comparison to those in the doxorubicin-induced cardiotoxicity group, the perindopril group rats exhibited decreased LVEDD and LVESD levels $(P<0.05)$ (Figure $2 b$ and $c$ ).

$\mathrm{H} \& \mathrm{E}$ staining demonstrated that rats in the doxorubicin-induced cardiotoxicity group displayed widely pathological damages, such as vacuolization and degeneration of cardiomyocytes, inflammatory cell infiltration and interstitial oedema, in comparison to those in the normal group (Supplementary Figure $1 a$ ). Electron microscopy analyses of heart sections from the doxorubicin-induced cardiotoxicity group confirmed random sarcomeres in cardiac muscles, vacuolar degeneration and swollen mitochondria, features not exhibited by the normal group rats. Notably, perindopril treatment ameliorated the histological damage induced by doxorubicin (Supplementary Figure $1 b$ ).

Rats in the doxorubicin-induced cardiotoxicity group had significantly lower LVFS and LVEF values than those in the normal group $(P<0.05)$, indicating impaired cardiac function in the former group. Compared to the cardiotoxicity group, rats in perindopril group showed lower LVEDD and LVESD values $(P<0.05)$ and increased LVEF as well as LVFS levels $(P<0.05)$ (Figure $3 a$ and $b$ ).

We evaluated the changes in glucose metabolism using ${ }^{18}$ F-FDG uptake. The rats did not exhibit signs of peritonitis after administration of $10 \%$ chloral hydrate. A significant decrease in myocardial ${ }^{18}$ F-FDG uptake and myocardial glucose metabolism was observed in rats from the doxorubicin-induced cardiotoxicity group in comparison to those from the normal group (SUV: $6.54 \pm 2.40$ versus $2.28 \pm 0.75, P<0.05$ ), which was markedly ameliorated by perindopril disposal (SUV: $3.85 \pm 1.23$ versus $2.28 \pm 0.75, P<0.05$ ) (Figure $3 c-f$ ).

BNP, CKMB and TnI of rats in the doxorubicininduced cardiotoxicity group had distinctly higher serum levels in comparison to those in the normal group $(P<0.001)$, indicating myocardial damage in the former group. However, these increases were significantly decreased by perindopril treatment $(P<0.05)$, demonstrating the improvement of myocardial damage following treatment (Supplementary Table 1).

Significant increase in the levels of angiotensinogen, AT1R, Ang II and PKC was observed in the doxorubicininduced cardiotoxicity group (Figure $4 a-d$ ). However, increase in Ang II, PKC and AT1R was significantly suppressed by perindopril treatment $(P<0.05)$. Also, perindopril had no effect on the expression of angiotensinogen in the doxorubicin-induced cardiotoxicity rats $(P>0.05)$ (Figure $4 a)$.

The ROS levels were distinctly higher in the doxorubicin-induced cardiotoxicity group $(P<0.05)$ compared to controls (Figure $4 e$ ). Nevertheless, the increase was significantly inhibited by perindopril disposal $(P<0.05)$ (Figure $4 e$ ).

At the mRNA level, HMGB 1 and NF- $\kappa \mathrm{B}$ expression was notably elevated in the doxorubicin-induced cardiotoxicity group than the normal group $(P<0.05)$ 

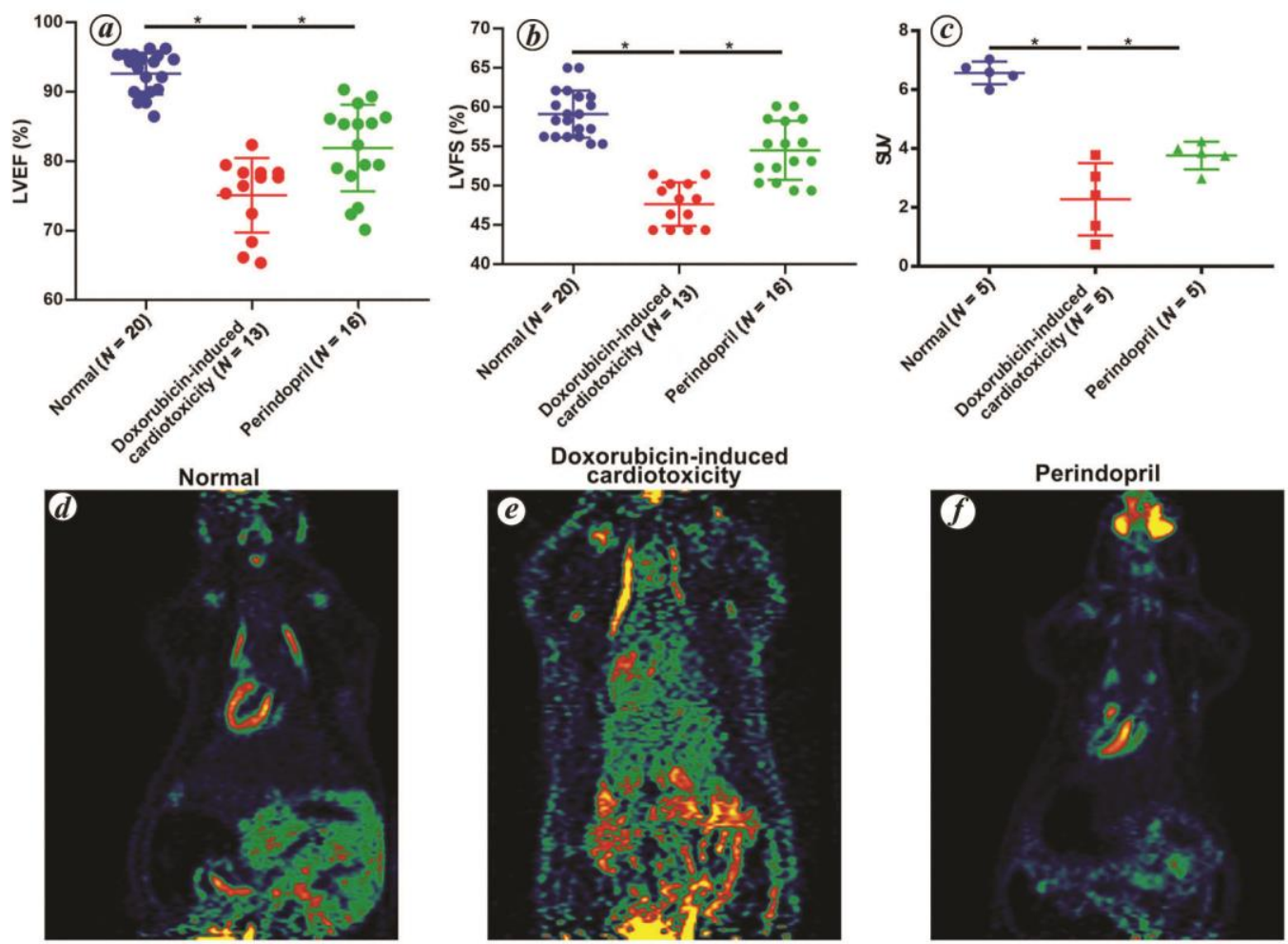

Figure 3. Cardiac function in doxorubicin-induced cardiotoxicity rats. $\boldsymbol{a}, \boldsymbol{b}$, Echocardiography in doxorubicininduced cardiotoxicity rats. Rats in the doxorubicin-induced cardiotoxicity group had significantly lower LVFS and LVEF levels in comparison to those in the normal group $(P<0.05)$. Compared to the doxorubicin-induced cardiotoxicity group, the perindopril group showed lower LVEDD and LVESD values $(P<0.05)$ and higher LVEF as well as LVFS levels $(P<0.05)$. $c$, SUV in the doxorubicin-induced cardiotoxicity group exhibited markedly higher levels in comparison to the normal group $(6.54 \pm 2.40$ versus $2.28 \pm 0.75 ; P<0.05)$. This increase was distinctly suppressed by perindopril treatment $(3.85 \pm 1.23$ versus $2.28 \pm 0.75 ; P<0.05)$. $d-f$, A significant decrease in myocardial ${ }^{18}$ F-FDG uptake and myocardial glucose metabolism was observed, as expected, for rats in the doxorubicin-induced cardiotoxicity group compared to those in the normal group, which was prominently ameliorated by perindopril treatment.

(Figure $4 f$ and $g$ ), which was distinctly inhibited by perindopril treatment $(P<0.05)$. Furthermore, HMGB1 and NF- $\kappa \mathrm{B}$ expression was significantly elevated in the doxorubicin-induced cardiotoxicity group $(P<0.05)$ than the normal group. Nevertheless, the increase was significantly inhibited due to perindopril disposal $(P<0.05)$ (Figure $4 h-j$ ).

The levels of TNF- $\alpha$, IL- 6 as well as IL- $1 \beta$ had distinctly increased in the doxorubicin-induced cardiotoxicity group in comparison to the normal group $(P<0.05)$, which was markedly ameliorated by perindopril disposal $(P<0.05)$ (Supplementary Figure 2).

A positive correlation was found between Ang II expression and ROS level in doxorubicin-induced cardiotoxicity rats $(P<0.0001, R=0.9703)$ (Figure $4 k)$. Also, Ang II expression had a positive correlation with HMGB1 expression $(P<0.0001, \quad R=0.9391)$ (Figure $4 k$ ). Furthermore, ROS level was positively correlated with HMGB1 expression $(P<0.0001, \quad R=0.9300)$ (Figure $4 k$ ).

In this study, we observed the effect of perindopril treatment on doxorubicin-induced cardiotoxicity and explored its potential molecular mechanism. The findings are as follows: (1) The expression levels of angiotensinogen, Ang II, AT1R, PKC and ROS were increased in doxorubicin-induced cardiotoxicity rats. (2) RAS activated HMGB1 and led to overactivation of NF- $\kappa \mathrm{B}$ in doxorubicin-induced cardiotoxicity rats, which regulated the production of a proinflammatory cascade and led to the subsequent dilation of the cardiac chamber and impaired cardiac function. (3) Blockade of Ang II by perindopril treatment ameliorated the effect induced by Ang II, demonstrating a key role concerning the ROSHMGB1 pathway for cardiomyocyte injury due to AngII. Our results elucidate a new molecular mechanism by which the increase in ROS secretion due to AngII results in HMGB1 overactivation, thereby regulating $\mathrm{NF}-\kappa \mathrm{B}$ to cause doxorubicin-induced cardiotoxicity. Data indicate that the above molecules may be promising therapeutic targets for doxorubicin-induced cardiotoxicity.

The doxorubicin-induced cardiotoxicity group exhibited impaired cardiac function. Both the LVEDD and LVESD values in the doxorubicin-induced cardiotoxicity group were notably elevated in comparison to those in the 

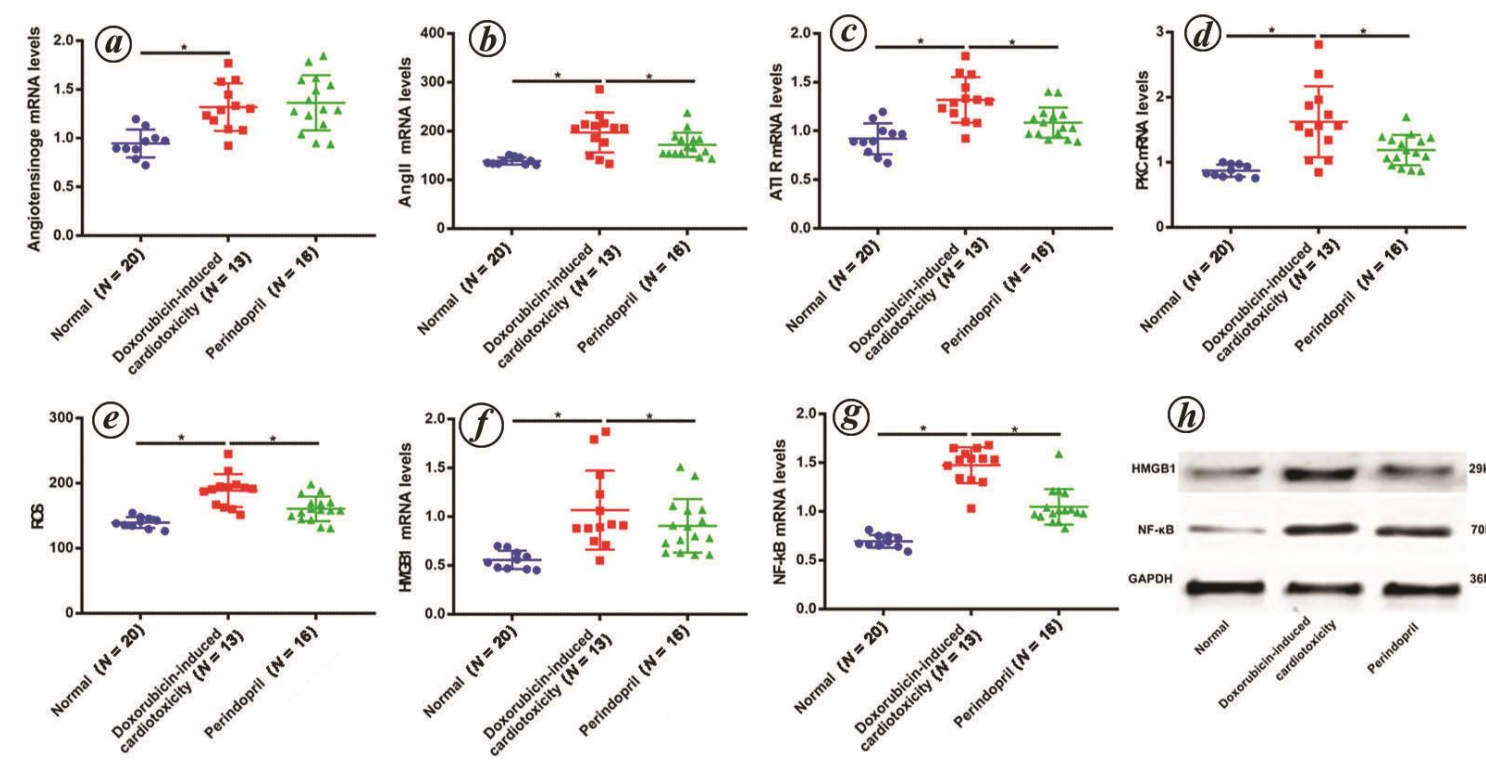

(b)
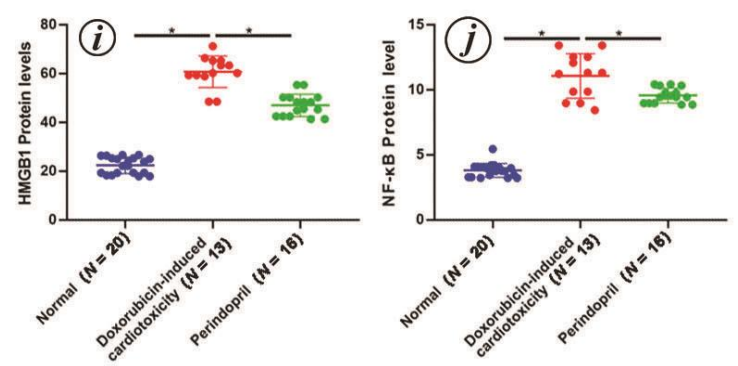

(1)
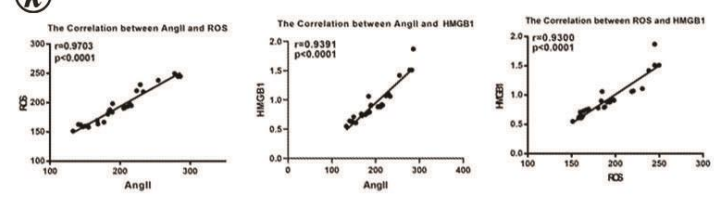

Figure 4. $\boldsymbol{a}-\boldsymbol{d}$, The myocardial mRNA expression of angiotensinogen, AT1R and PKC in the three groups and myocardial protein expression of Ang II were conspicuously elevated in the doxorubicin-induced cardiotoxicity group in comparison to the normal group $(P<0.05)$, which decreased due to perindopril disposal $(P<0.05) . \boldsymbol{e}, \mathrm{ROS}$ in the doxorubicin-induced cardiotoxicity group exhibited significantly higher level in comparison to the normal group $(P<0.05)$, which was significantly suppressed due to perindopril treatment $(P<0.05) . f-j$, At the protein and mRNA levels, HMGB1 and NF- $\kappa$ B expression was prominently elevated in the doxorubicin-induced cardiotoxicity group than the normal group $(P<0.05)$, and decreased due to perindopril treatment $(P<0.05) . \boldsymbol{k}$, ROS concentration and Ang II expression were positively correlated in the doxorubicin-induced cardiotoxicity group $(P<0.0001$ and $R=0.9703)$. Ang II and HMGB1 were positively correlated $(P<0.0001$ and $R=0.9391)$. ROS and HMGB1 were also positively correlated $(P<0.0001$ and $R=0.9300)$.

normal group, while the LVEF and LVFS values had decreased. The impaired myocardium was further confirmed by the increased circulating levels of BNP, CKMB and TnI, which are biomarkers of cardiac injury ${ }^{23,24}$. PET imaging of glucose metabolism can be used to evaluate myocardial viability following myocardial infarction in patients with coronary heart illness ${ }^{19}$. Here, ${ }^{18}$ F-FDG PET myocardial metabolic imaging was utilized to evaluate myocardial viability in doxorubicin-induced cardiotoxicity rats. As expected, the SUVs had decreased significantly in the doxorubicin-induced cardiotoxicity rats compared with the normal rats due to the dilated cardiac chamber size and impaired cardiac function. The doxorubicininduced cardiotoxicity rats were treated with perindopril, resulting in a distinct elevation in SUVs in them in comparison to the doxorubicin-induced cardiotoxicity rats, and the cardiac structure and function were improved. These results illustrate that the myocardial injury and enlargement of the cardiac cavity were improved.
Ventricular remodelling is an important pathophysiological aspect of doxorubicin-induced cardiotoxicity, which is associated with its development ${ }^{6}$. RAAS is involved in doxorubicin-induced cardiotoxicity-related ventricular remodelling in many ways ${ }^{6}$. Ang II has a relationship with occurrences of heart failure, related to inflammatory response as well as interstitial fibrosis ${ }^{25}$. Here, angiotensinogen, Ang II, and PKC expressions were elevated in the doxorubicin-induced cardiotoxicity rats. However, the increase in Ang II and PKC was significantly suppressed by perindopril disposal. Ang II promotes the transition of myocardium from a contractile to a synthetic phenotype, slows down the maximum myocardial shortening speed and reduces myocardial tension, thus leading to a decrease in myocardial contractility. However, Ang II can increase the synthesis of collagen in the myocardial interstitium, change the composition of the extracellular matrix and influence myocardial interstitial fibrosis ${ }^{26,27}$. PKC is a key component for myocardial 


\section{RESEARCH COMMUNICATIONS}

fibrosis. Ang II promotes myocardial fibrosis via integrin $\beta 1$, and $\mathrm{PKC}$ is a specific signalling pathway that activates integrin $\beta 1$ (ref. 14).

Previous in vivo and in vitro studies have suggested that Ang II binds to AT1R, activates NOX and promotes ROS production in the mitochondria of cardiomyocytes and endothelial cells, while blocking Ang II or AT1R can reverse $\operatorname{ROS}^{8,9}$. Here, ROS level was elevated in the doxorubicin-induced cardiotoxicity rats. A positive correlation was observed between Ang II expression and ROS concentration in doxorubicin-induced cardiotoxicity rats, and the increased ROS level was ameliorated by perindopril treatment. ROS can destroy the integrity of the myocardial cell membrane and lead to myocardial cell death. ROS can also damage myocardial cells, causing mitochondrial swelling, vacuole formation and even disintegration. This phenomenon was also confirmed by our histopathological and ${ }^{18}$ F-FDG PET myocardial metabolic imaging results. Furthermore, ROS can cause sarcoplasmic reticulum dysfunction, reducing $\mathrm{Ca}^{2+}$ uptake and affecting excitation-contraction coupling. Therefore, ROS impair myocardial function, affect myocardial contraction and diastolic function, and ultimately lead to the development of doxorubicin-induced cardiotoxicity and heart failure ${ }^{28}$.

Moreover, increase in inflammation and fibrosis is related to the pathogenesis of heart failure ${ }^{10,29}$, and the nonhistone nuclear factor HMGB1 shows proinflammatory attributes ${ }^{30}$. When cells are activated, they excrete HMGB1. However, if the cells are damaged, they can produce HMGB1 extracellularly to serve as a facilitator (secreted by macrophages) as well as promoter (released by necrotic cells) of inflammation ${ }^{31,32}$. Previous studies have found that HMGB1 and its inflammatory signalling pathway HMGB1-TLR4/RAGE-NF- $\kappa$ B-cytokines are highly expressed in doxorubicin-induced cardiotoxicity rats and associated with the cardiac chamber size and cardiac function, suggesting that the HMGB1-TLR4/ RAGE-NF- $\kappa$ B-cytokine axis may be a pathophysiological mechanism underlying the development of doxorubicin-induced cardiotoxicity ${ }^{11}$. An in vitro study indicates that Ang II can increase HMGB1 expression, leading to over activation of TLR4 and thereby regulating the production of cytokines induced by $\mathrm{NF}-\kappa \mathrm{B}$ (ref. 8). One of the major mechanisms underlying doxorubicin-induced cardiomyopathy is increased oxidative stress $^{22}$, which results in elevated apoptosis and inflammatory responses. Other studies have reported that ROS can promote HMGB1 release through macrophages and monocytes ${ }^{16}$. Moreover, oxidative stress modulates the excretion of HMGB1 in atrial fibrillation patients ${ }^{17}$. However, in doxorubicin-induced cardiotoxicity, whether the high expression of HMGB1 is a result of the interaction between Ang II and ROS remains unclear.

The results of this study show increased levels of Ang II, ROS, HMGB1, NF- $\mathrm{KB}$ and subsequent proinflamma- tory cytokines like TNF- $\alpha$, IL- 6 as well as IL- $1 \beta$ in doxorubicin-induced cardiotoxicity rats. Furthermore, Ang II, ROS and HMGB1 positively correlate with each other. The release of HMGB1 is promoted by Ang II and ROS to activate the signalling pathway for downstream inflammation. Proinflammatory cytokines can directly induce myocardial contractility and promote cardiomyocyte hypertrophy and progressive apoptosis, leading to myocardial remodelling; these cytokines can also induce oxidative stress and reduce the myocardial cellular antioxidant capacity ${ }^{33}$. This release also causes the consequent enlargement of the cardiac cavity and cardiac dysfunction. We also treated the doxorubicin-induced cardiotoxicity rats with the angiotensin-converting enzyme inhibitor perindopril, resulting in inhibition of Ang II and decrease in ROS and HMGB levels. Consequently, the SUVs distinctly increased in the perindopril rats compared with those treated with doxorubicin; furthermore, the structure was improved. These results illustrate that the myocardial injury and enlargement of the cardiac cavity are improved, which further highlights the relationship among the three factors.

This study has identified the relationships among Ang, ROS and HMGB for doxorubicin-induced cardiotoxicity rats. Future studies involving in vitro assays using cellular models of doxorubicin-induced myocardial damage are required for in-depth verification of the mechanisms.

Competing interest: The authors declare no conflict of interest.

1. Zhang, S., Liu, X., Bawa-Khalfe, T., Lu, L. S., Lyu, Y. L., Liu, L. F. and Yeh, E. T., Identification of the molecular basis of doxorubicin-induced cardiotoxicity. Nature Med., 2012, 18, 16391642 .

2. Songbo, M., Lang, H., Xinyong, C., Bin, X., Ping, Z. and Liang, S., Oxidative stress injury in doxorubicin-induced cardiotoxicity. Toxicol. Lett., 2019, 307, 41-48.

3. Koleini, N., Nickel, B. E., Edel, A. L., Fandrich, R. R., Ravandi, A. and Kardami, E., Oxidized phospholipids in doxorubicininduced cardiotoxicity. Chem. Biol. Interact., 2019, 303, 35-39.

4. Wenningmann, N., Knapp, M., Ande, A., Vaidya, T. R. and AitOudhia, S., Insights into doxorubicin-induced cardiotoxicity: molecular mechanisms, preventive strategies, and early monitoring. Mol. Pharmacol., 2019, 96, 219-232.

5. Shabalala, S., Muller, C. J. F., Louw, J. and Johnson, R., Polyphenols, autophagy and doxorubicin-induced cardiotoxicity. Life Sci., 2017, 180, 160-170.

6. Cody, R. J., Haas, G. J., Binkley, P. F., Capers, Q. and Kelley, R., Plasma endothelin correlates with the extent of pulmonary hypertension in patients with chronic congestive heart failure. Circulation, 1992, 85, 504-509.

7. Mackiewicz, U. et al., Preserved cardiomyocyte function and altered desmin pattern in transgenic mouse model of dilated cardiomyopathy. J. Mol. Cell. Cardiol., 2012, 52, 978-987.

8. Privratsky, J. R., Wold, L. E., Sowers, J. R., Quinn, M. T. and Ren, J., At1 blockade prevents glucose-induced cardiac dysfunction in ventricular myocytes: role of the AT1 receptor and NADPH oxidase. Hypertension, 2003, 42, 206-212. 


\section{RESEARCH COMMUNICATIONS}

9. Zhang, A., Wang, M. and Zhuo, P., Unc-51 like autophagy activating kinase 1 accelerates angiotensin II-induced cardiac hypertrophy through promoting oxidative stress regulated by NRF-2/HO-1 pathway. Biochem. Biophys. Res. Commun., 2019, 509, 32-39.

10. Volz, H. C. et al., HMGB1 is an independent predictor of death and heart transplantation in heart failure. Clin. Res. Cardiol., 2012, 101, 427-435.

11. Shen Lijuan, L. S., Yonghua, Z., Qingmin, X., Lan, L. and Chungang, Z., Expression and effect of HMGB1 and its inflammatory signaling pathway in rat model of DCM. Chongqin Yixue, 2017, 46, 1457-1462.

12. Rose, N. R., Myocarditis: infection versus autoimmunity. J. Clin. Immunol., 2009, 29, 730-737.

13. Eriksson, U., Kurrer, M. O., Schmitz, N., Marsch, S. C., Fontana, A., Eugster, H. P. and Kopf, M., Interleukin-6-deficient mice resist development of autoimmune myocarditis associated with impaired upregulation of complement C3. Circulation, 2003, 107, 320-325.

14. de Souza, A. W., Westra, J., Limburg, P. C., Bij1, M. and Kallenberg, C. G., HMGB1 in vascular diseases: its role in vascular inflammation and atherosclerosis. Autoimmunity Rev., 2012, 11, 909-917.

15. Nair, A. R., Ebenezer, P. J., Saini, Y. and Francis, J., Angiotensin II-induced hypertensive renal inflammation is mediated through HMGB1-TLR4 signaling in rat tubulo-epithelial cells. Exp. Cell Res., 2015, 335, 238-247.

16. Tang, D., Shi, Y., Kang, R., Li, T., Xiao, W., Wang, H. and Xiao, $\mathrm{X}$., Hydrogen peroxide stimulates macrophages and monocytes to actively release HMGB1. J. Leukocyte Biol., 2007, 81, 741-747.

17. Wu, Y., Zhang, K., Zhao, L., Guo, J., Hu, X. and Chen, Z., Increased serum HMGB1 is related to oxidative stress in patients with atrial fibrillation. J. Int. Med. Res., 2013, 41, 1796-1802.

18. Shen, L. J., Lu, S., Zhou, Y. H., Li, L., Xing, Q. M. and Xu, Y. L., Developing a rat model of dilated cardiomyopathy with improved survival. J. Zhejiang Univ. Sci. B, 2016, 17, 975-983.

19. Handa, N., Magata, Y., Mukai, T., Nishina, T., Konishi, J. and Komeda, M., Quantitative FDG-uptake by positron emission tomography in progressive hypertrophy of rat hearts in vivo. Ann. Nucl. Med., 2007, 21, 569-576.

20. Stegger, L. et al., Monitoring left ventricular dilation in mice with pet. J. Nuclear Med., 2005, 46, 1516-1521.

21. Gava, F. N. et al., Doxorubicin induced dilated cardiomyopathy in a rabbit model: an update. Res. Vet. Sci., 2013, 94, 115-121.

22. Lu, D. et al., Knockdown of cytochrome p450 2E1 inhibits oxidative stress and apoptosis in the cTnT(R141W) dilated cardiomyopathy transgenic mice. Hypertension, 2012, 60, 81-89.

23. Iqbal, N., Wentworth, B., Choudhary, R., Landa Ade, L., Kipper, B., Fard, A. and Maisel, A. S., Cardiac biomarkers: new tools for heart failure management. Cardiovasc. Diagn. Ther., 2012, 2 147-164.

24. Anand, I. S. et al., C-reactive protein in heart failure: prognostic value and the effect of valsartan. Circulation, 2005, 112, 14281434.

25. Okabe, K., Teneligliptin attenuated AngII-induced cardiac hypertrophy by inhibiting NOX4-HDAC4 axis. J. Card. Fail., 2017, 23, S56.

26. Willenheimer, R., Left ventricular remodelling and dysfunction. Can the process be prevented? Int. J. Cardiol., 2000, 72, 143150 .

27. Chintalgattu, V. and Katwa, L. C., Role of protein kinase C-delta in angiotensin II induced cardiac fibrosis. Biochem. Biophys. Res. Commun., 2009, 386, 612-616.

28. Hantson, P., Mechanisms of toxic cardiomyopathy. Clin. Toxicol., 2019, 57, 1-9.

29. Li, H. et al., Regulator of $\mathrm{G}$ protein signaling 5 protects against cardiac hypertrophy and fibrosis during biomechanical stress of pressure overload. Proc. Natl. Acad. Sci. USA, 2010, 107, 1381813823.

30. Ulloa, L. and Messmer, D., High-mobility group box 1 (HMGB1) protein: Friend and foe. Cytokine Growth Factor Rev., 2006, 17, 189-201.

31. Scaffidi, P., Misteli, T. and Bianchi, M. E., Release of chromatin protein HMGB1 by necrotic cells triggers inflammation. Nature, 2002, 418, 191-195.

32. Vande Walle, L., Kanneganti, T. D. and Lamkanfi, M., HMGB1 release by inflammasomes. Virulence, 2011, 2, 162-165.

33. Haudek, S. B., Taffet, G. E., Schneider, M. D. and Mann, D. L., TNF provokes cardiomyocyte apoptosis and cardiac remodeling through activation of multiple cell death pathways. J. Clin. Invest., 2007, 117, 2692-2701.

ACKNOWLEDGMENTS. This research was supported by the Jiangsu Province TCM Leading Talents Training Object Project and the Wuxi Medical Outstanding Talents Cultivation Project (JCRCPY005), China. Animal protocols were approved by the Animal Care and Use Committee of the Institute of Key Laboratory on Technology for Parasitic Disease Prevention and Control, Ministry of Health, China. Animal experiments were in compliance with the Guide for the Care and Use of Laboratory Animals by the Chinese Ministry of Science and Technology. The datasets generated and analysed in the present study are available with the corresponding author upon request.

Received 17 March 2020; revised accepted 19 September 2020

doi: $10.18520 / \mathrm{cs} / \mathrm{v} 119 / \mathrm{i} 11 / 1838-1845$

\section{Effect of water quality on caddisflies (Trichoptera) in Kallada river, Kerala, India}

\section{Dani Benchamin*, R. Sreejai and Beena S. Kurup}

Zoology Research Centre, St Stephen's College, University of Kerala, Pathanapuram 689 695, India

The caddisflies (Trichoptera) constitute a significant water quality marker group because of their taxonomic richness, ecological diversity, and abundance in virtually all freshwater ecosystems. In this study, caddisflies were collected for an evaluation of the water quality in Kallada river, South, India. Samples collected during the pre-monsoon season were identified at the family level. In total, more than 4500 specimens were identified as belonging to five different taxa. These sensitive species have typically been restricted to non-polluted water bodies. The dominant family at stations S1, S2, S3 and S4 was determined to

*For correspondence. (e-mail: dani.gethsemane@gmail.com) 